\title{
Structural Frequency Response Function Prediction and Experimental Validation between Aircraft Engine Mount and Pylon
}

\author{
Junwei $\mathrm{Xu}^{1+}$, Luyao Ge ${ }^{1}$, Feng Han ${ }^{1}$ and Huayong Zhao ${ }^{1}$ \\ ${ }^{1}$ Functional Structure Department, Shanghai Aircraft Design and Research Institute, Shanghai, China, \\ 201210
}

\begin{abstract}
Structure frequency response function (FRF) prediction and validation are necessary for aircraft noise and vibration engineering. With the frequency response functions (FRFs), the vibration transmission characteristic can be evaluated by engineer so that potential structure resonance behavior can be found at specific frequencies. In this paper, the concerned vibration frequencies come from the engine low pressure rotor and high pressure rotor during engine operation in cruise condition. The finite elements model of engine mount, pylon and pylon spar with fuselage was established and the frequency response functions between the engine mount excitation location and the pylon response locations were calculated. Then hammer method was used to acquire actual frequency response functions to validate the calculation results. The comparison results show that it was in good agreement between the calculated results and experimental results. From these frequency response functions, it could be evaluated that there was no resonant characteristics for the engine mount, pylon and pylon spar with fuselage structures at the concerned frequencies and there was no risk for the structure-borne noise problem caused by engines.
\end{abstract}

Keywords: FRF calculation, FRF testing, structural mode, hammer method, engine vibration.

\section{Introduction}

For the commercial aircraft, the noise and vibration are the key indicators for comfort evaluation. Typically, modern commercial aircraft adopts turbofan engines as it power and this kind of engine has two shafts, one is the low pressure rotor (N1) and the other is high pressure rotor (N2). For these two rotors, absolute balance is impossible in the actual situation so that the dynamic force will be produced during the operation condition[1]. This kind of dynamic forces will be transferred to the airframe structures through engine mount and pylon, further, airframe vibration and structure-borne noise in the passenger cabin will appear. During the engine operation, the two shafts will produce harmonic vibration at specific frequencies related to their revolution speeds [2]. The fundamental frequency of the harmonic vibration can be calculated by Eq.(1).

$$
f=\frac{N}{60}
$$

where $f$ denotes the fundamental frequency of the harmonic vibration and the unit is $\mathrm{Hz} ; \mathrm{N}$ denotes the revolution speed of N1 or N2 rotor, the unit is Revolutions Per Minute (RPM). For the aircraft, the engine $\mathrm{N} 1$ and $\mathrm{N} 2$ revolution speeds are often steady during the cruise condition and this is also the most concerned condition for noise and vibration engineering.

In this paper, one kind of engine fuselage mounted aircraft is introduced and the engine N1 and N2 revolution speed is 5280 RPM and 16190 RPM during cruise condition. Based on Eq.(1), the fundamental frequency of the $\mathrm{N} 1$ and $\mathrm{N} 2$ vibration is $88 \mathrm{~Hz}$ and $270 \mathrm{~Hz}$, respectively. As the main source of noise and vibration in the cabin, aircraft engines and their related structures such as mount, pylon and pylon spar

\footnotetext{
+ Corresponding author. Tel.: +86-153 1618 9139; fax: n/a.

E-mail address:xujunwei@comac.cc.
} 
should be carefully designed so that they have no resonance effect to amplify the vibration from engine at the fundamental frequency of the N1 and N2.

The way to evaluate the structure vibration transfer characteristics is through the structural FRFs of engine mount, pylon and pylon spar with fuselage [2]. In this paper, the basic theory for the FRF was introduced and based on this theory, the engine mount, pylon and pylon spar with fuselage models were established by finite elements. With these finite elements models, the structural modes were calculated and further the FRFs between excitation location on the mount and the response locations on the pylon joint and pylon spar with fuselage were calculated. In order to validate these prediction FRFs, hammer method was used as the experimental approach for validation. At last, compared with these calculated FRFs and experimental FRFs, it shown that it was in good agreementt between the calculated results and experimental results. Furthermore, it was confirmed that there was no resonant characteristics for the airframe structure itself at the concerned frequency of $86 \mathrm{~Hz}$ and $270 \mathrm{~Hz}$ and it may has no risk for the structure-borne noise caused by engine vibration.

\section{Theory for Structural FRF of Multiple Degrees of Freedom System}

In general, the real structure is a multiple degrees of freedom system and the differential equation of its vibration [3] can be described as

$$
\boldsymbol{M} \ddot{\boldsymbol{x}}+(\boldsymbol{K}+j \boldsymbol{G}) \boldsymbol{x}=\boldsymbol{F} \boldsymbol{e}^{j \omega t}
$$

where $\boldsymbol{M}, \boldsymbol{K}$ and $\boldsymbol{G}$ denote the $n \times n$ mass, stiffness and damping matrices, respectively; $\boldsymbol{F} \boldsymbol{e}^{j \omega t}$ is the $n \times 1$ excitation force vector; $\ddot{\boldsymbol{x}}$ and $\boldsymbol{x}$ are the $n \times 1$ vectors of acceleration or displacement, respectively.

Assuming the right side of Eq.(2) is zero, and the homogeneous equation of Eq.(2) is

$$
\boldsymbol{M} \ddot{\boldsymbol{x}}+(\boldsymbol{K}+j \boldsymbol{G}) \boldsymbol{x}=\mathbf{0}
$$

Defining the special solution for Eq.(3) is $\boldsymbol{x}=\boldsymbol{\psi} e^{\lambda t}$ and Eq.(3) can be described as

$$
\left(\lambda^{2} \boldsymbol{M}+\boldsymbol{K}+j \boldsymbol{G}\right) \boldsymbol{\psi}=\mathbf{0}
$$

where $\lambda^{2}$ is the eigenvalues and $\psi$ is the eigenvectors of Eq.(4).

The characteristic equation of Eq.(4) can be obtained as

$$
\left|\lambda^{2} \boldsymbol{M}+\boldsymbol{K}+j \boldsymbol{G}\right|=\mathbf{0}
$$

Eq.(5) can be solved and obtained $n$ eigenvalues $\lambda^{2}$ with $n$ eigenvectors $\psi$. The complex eigenvector $\boldsymbol{\psi}$ is known as a modal matrix and can be described as

$$
\psi=\left[\begin{array}{llll}
\psi_{1} & \psi_{2} & \cdots & \psi_{n}
\end{array}\right]
$$

Complex modal parameters for multiple degrees of freedom system can be described as

$$
\left.\begin{array}{rl}
m_{m i} & =\boldsymbol{\psi}_{i}^{H} \boldsymbol{M} \boldsymbol{\psi}_{i} \\
k_{m i} & =\boldsymbol{\psi}_{i}^{H} \boldsymbol{K} \boldsymbol{\psi}_{i} \\
g_{m i} & =\boldsymbol{\psi}_{i}^{H} \boldsymbol{G} \boldsymbol{\psi}_{i} \\
\omega_{m i}^{2} & =\frac{k_{m i}}{m_{m i}} \\
\eta_{m i} & =\frac{g_{m i}}{k_{m i}}
\end{array}\right\}
$$

where $m_{m i}, k_{m i}, g_{m i}, \omega_{m i}$ and $\eta_{m i}$ denote the complex characteristic mass, complex characteristic stiffness, complex characteristic damping, complex modal frequency and complex loss factor of structures belong to structural damping system, respectively. And the eigenvalues $\lambda^{2}$ can be written as

$$
\lambda_{i}^{2}=-\frac{k_{D i}}{m_{D i}}-j \frac{g_{D i}}{m_{D i}}=-\omega_{m i}^{2}\left(1+j \eta_{m i}\right)
$$

Eq.(7) and Eq.(8) reflect the inherent characteristics of the vibration system [3,4]. 
The frequency response function matrix of multiple degrees of freedom system can be expressed as

$$
\boldsymbol{H}(\omega)=\left(\boldsymbol{K}-\omega^{2} \boldsymbol{M}+j \boldsymbol{G}\right)^{-1}
$$

where $\boldsymbol{H}$ is the frequency response function matrix; $\boldsymbol{M}, \boldsymbol{K}$ and $\boldsymbol{G}$ denote mass matrix, stiffness matrix and damping matrix, respectively. Because of the orthogonality of the eigenvectors $\psi_{i}$, the complex modal coordinate system and physical coordinate system can be transferred by

$$
\boldsymbol{x}=\psi \boldsymbol{y}
$$

where $\boldsymbol{y}$ is the coordinate vectors of $\boldsymbol{x}$ in complex modes. Using the relationship of Eq.(10), the Eq.(2) can be described as

$$
\operatorname{diag}\left[m_{D i}\right] \ddot{\boldsymbol{y}}+\operatorname{diag}\left[k_{D i}+j g_{D i}\right] \boldsymbol{y}=\boldsymbol{\psi}^{T} \boldsymbol{F} e^{j \omega t}
$$

And the modal expansion form of frequency response functions (FRFs) can be obtained as

$$
\boldsymbol{H}(\omega)=\sum_{i=1}^{n} \frac{\boldsymbol{\psi}_{i} \boldsymbol{\psi}_{i}^{T}}{k_{D i}-\omega^{2} m_{D i}+j g_{D i}}
$$

The frequency response functions (FRFs) also can be expressed with complex modal parameters Eq.(7) as Eq.(13)

$$
\boldsymbol{H}(\omega)=\sum_{i=1}^{n} \frac{m_{m i}}{m_{D i}} \cdot \frac{\boldsymbol{\psi}_{i} \boldsymbol{\psi}_{i}^{T}}{k_{m i}-\omega^{2} m_{m i}+j g_{m i}}
$$

With the Eq.(13), the FRFs of multiple degrees of freedom system can be calculated from modal matrix and related modal parameters of vibration system. The FRF matrix reflects the attributes of the structure itself and it does not relate to the external excitations [3,4]. With the development of computational technology, commercial engineering software has the ability to compute the complex modes of large-scale structural system by finite element method and then acquires FRFs by defining the excitation and response locations[5,6].

\section{The Experimental Approach for Frequency Response Function}

A variety of FFT-based digital spectrum analyzers have the function to compute frequency response functions between the excitation point and response location based on the force and acceleration signal acquisition. For a multiple degrees of freedom system, the response matrix and excitation matrix [7] can be defined in the frequency domain as

$$
\boldsymbol{G}_{f x}(\omega)=\boldsymbol{H}(\omega) \boldsymbol{G}_{f f}(\omega)
$$

where $\boldsymbol{G}_{f f}(\omega)$ denote the auto-power spectrum matrix of excitation force ; $\boldsymbol{G}_{f x}(\omega)$ denote the crossspectrum matrix between the excitation force and response caused by this force at location $x . \boldsymbol{G}_{f f}(\omega)$ and $\boldsymbol{G}_{f x}(\omega)$ can be expressed as

$$
\begin{array}{r}
G_{f f}(\omega)=\left[\begin{array}{cccc}
G_{f 1 f 1}(\omega) & G_{f 2 f 1}(\omega) & \cdots & G_{f n f 1}(\omega) \\
G_{f 1 f 2}(\omega) & G_{f 2 f 2}(\omega) & \cdots & G_{f n f 2}(\omega) \\
\vdots & \vdots & & \vdots \\
G_{f 1 f n}(\omega) & G_{f 2 f n}(\omega) & \cdots & G_{f n f n}(\omega)
\end{array}\right] \\
G_{f x}(\omega)=\left[\begin{array}{cccc}
G_{f 1 x 1}(\omega) & G_{f 2 x 1}(\omega) & \cdots & G_{f n x 1}(\omega) \\
G_{f 1 x 2}(\omega) & G_{f 2 x 2}(\omega) & \cdots & G_{f n x 2}(\omega) \\
\vdots & \vdots & & \vdots \\
G_{f 1 x n}(\omega) & G_{f 2 x n}(\omega) & \cdots & G_{f n x n}(\omega)
\end{array}\right]
\end{array}
$$

With Eq.(14), the frequency response function matrix can be described as

$$
\boldsymbol{H}(\omega)=\frac{\boldsymbol{G}_{f x}(\omega)}{\boldsymbol{G}_{f f}(\omega)}
$$




\section{FRFs Prediction for Engine Mount and Pylon STRUCTURES}

\subsection{Finite element model}

A finite element model which including engine mount, pylon and nearest fuselage was established as shown in Fig. 1. The engine mount and pylon were created by 3D elements with the material of titanium alloy, and they were connected by bolts connections. The fuselage was created by $2 \mathrm{D}$ elements with the material of aluminium alloy and it was connected with pylon by rivets. In this model, defining the heading direction as $\mathrm{X}$ axis, lateral direction as $\mathrm{Y}$ axis and vertical direction as $\mathrm{Z}$ axis.

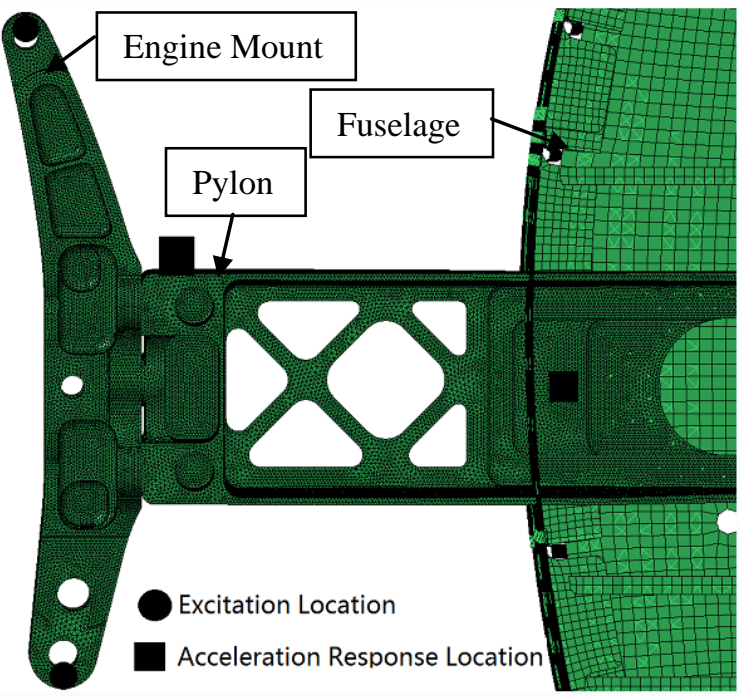

Fig. 1: Finite element model of engine mount, pylon and fuselage

In order to acquire a more accurate stimulation, a lumped mass unit equals to $4900 \mathrm{lb}$ was exerted on the upper and lower bolt holes of engine mount as the weight of engine and the edges of fuselage section were clamped as the constraint conditions.

\subsection{Structural modes calculation}

The finite element model established in Section 4.1 was submitted to the server for the structure modes calculation and the upper limit of modal frequency was set to $600 \mathrm{~Hz}$. Finally, 310 modal orders were captured by solver within the frequency scope of $0 \mathrm{~Hz}$ to $600 \mathrm{~Hz}$. Fig. 2 and Fig. 3 shown the structure modal shape colormaps at the 7 th modal frequency $(57.28 \mathrm{~Hz})$ and $302 \mathrm{nd}$ modal frequency $(587.22 \mathrm{~Hz})$.

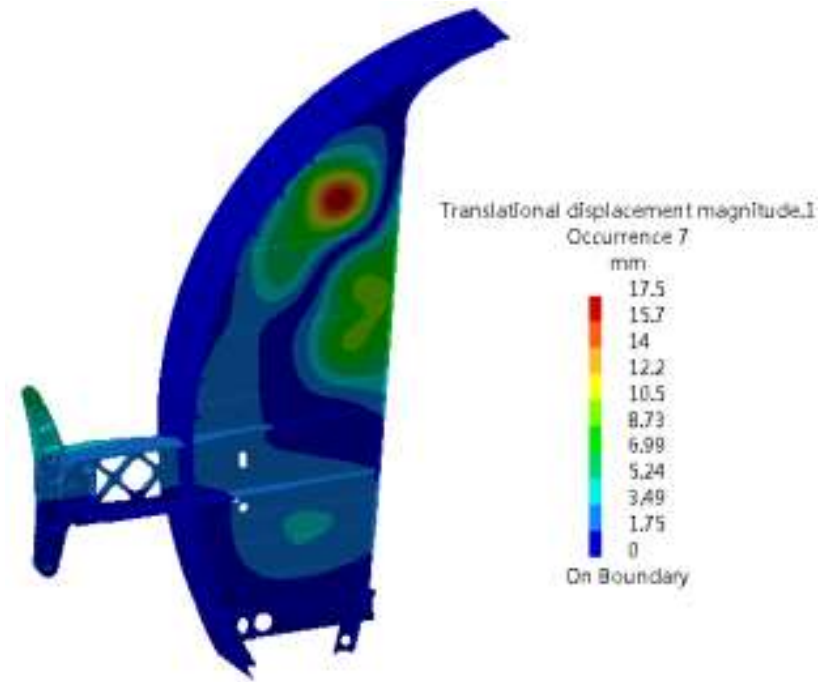

Fig. 2: The 7th modal shape colormap $(57.28 \mathrm{~Hz})$

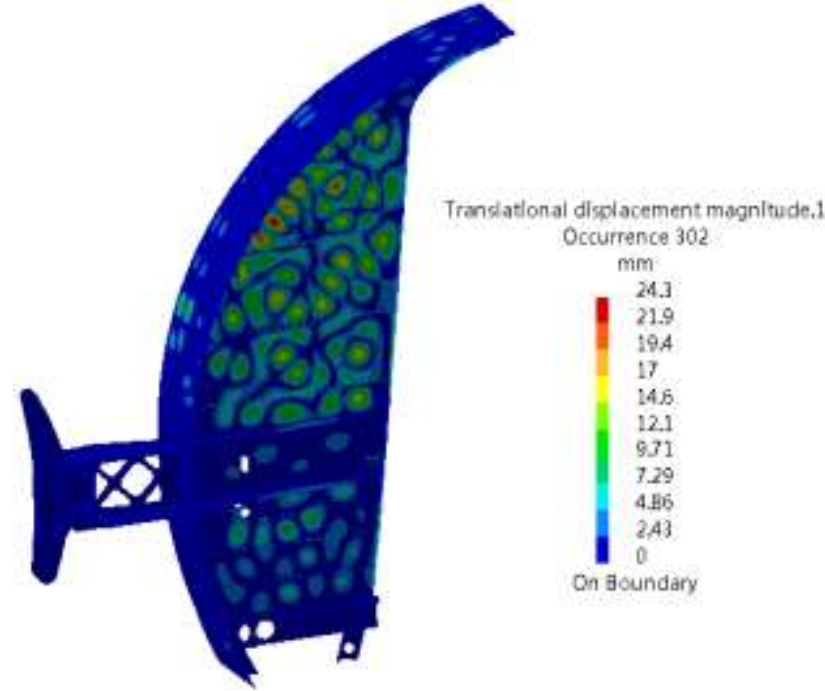

Fig. 3: The 302nd modal shape colormap $(587.22 \mathrm{~Hz})$

\subsection{Frequency response function calculation results}

With the modal results calculated by finite element solver, the FRFs between the excitation locations and acceleration response locations can be computed by the basic theory in Section 2. The Fig. 4 to Fig.6 show 
the FRFs between the lower excitation location with pylon joint acceleration response location and pylon spar acceleration response location. The Fig. 7 to Fig.9 show the FRFs between the upper excitation location with pylon joint acceleration response location and pylon spar acceleration response location.

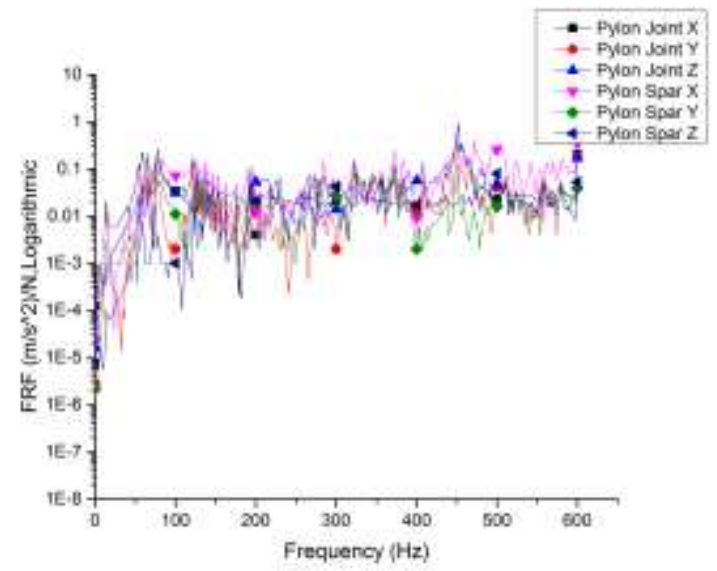

Fig. 4: FRF of lower excitation location at $\mathrm{X}$ axis

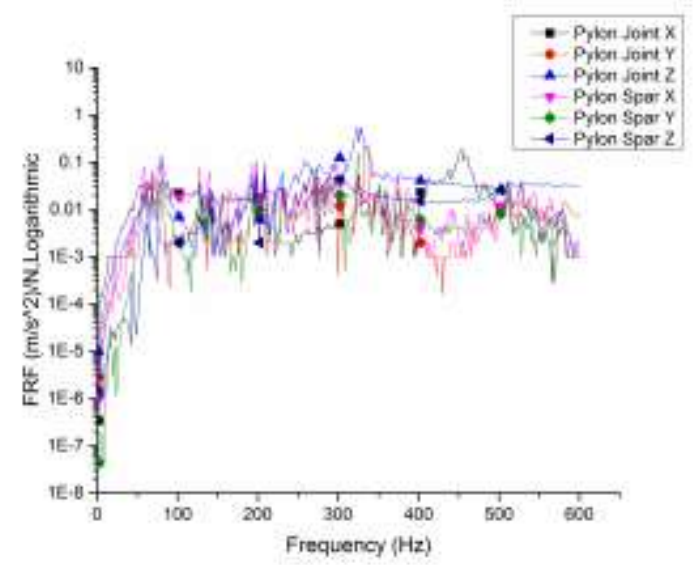

Fig. 6: FRF of lower excitation location at $\mathrm{Z}$ axis

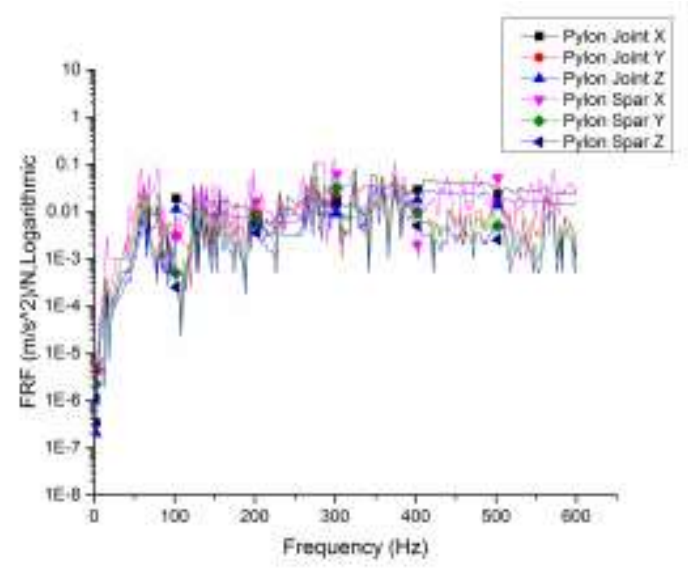

Fig. 8: FRF of upper excitation location at $\mathrm{Y}$ axis

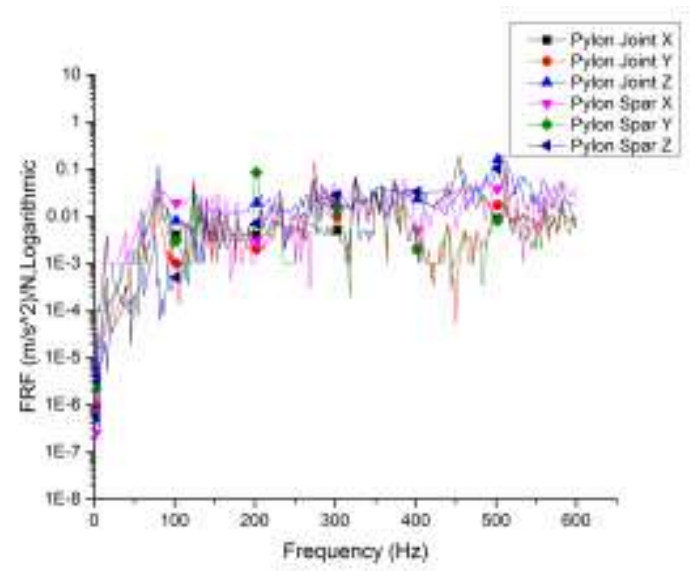

Fig. 5: FRF of lower excitation location at $\mathrm{Y}$ axis

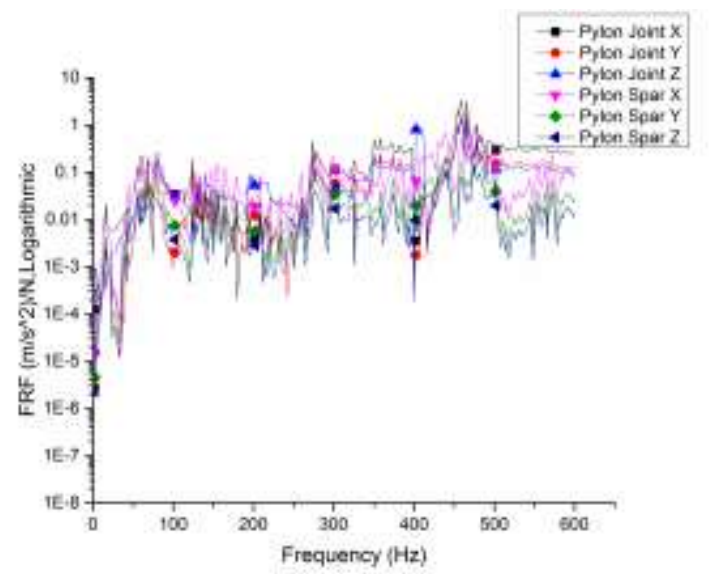

Fig. 7: FRF of upper excitation location at $\mathrm{X}$ axis

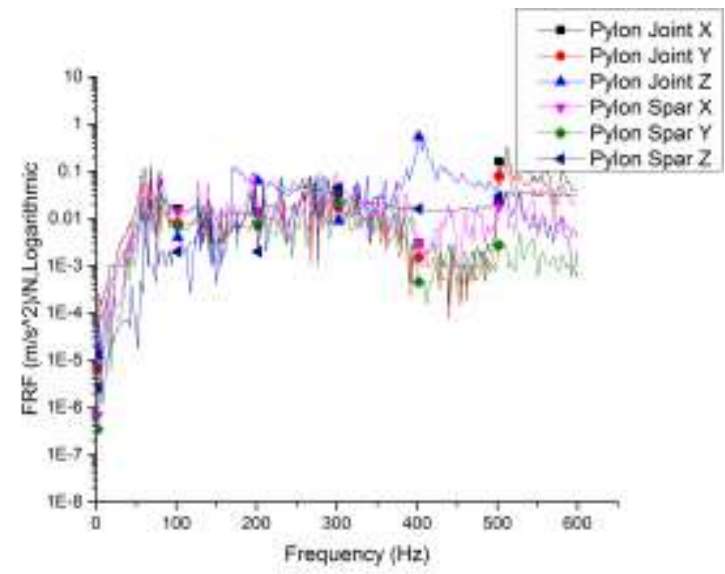

Fig. 9: FRF of upper excitation location at $Z$ axis

From Fig. 4 to Fig. 9, it seems that there isn't any "peak area" for the fundamental frequency of N1 $88 \mathrm{~Hz}$ and $\mathrm{N} 2270 \mathrm{~Hz}$, but for the upper excitation location at X direction, the FRF value is actually very high from $400 \mathrm{~Hz}$ to $500 \mathrm{~Hz}$ and this characteristic should be paid attention by the engineer. 


\section{Experimental Test for Engine Mount and Pylon Frequency Response Function}

\subsection{Experimental method}

In general, there are two methods for the structural FRF test. One is the hammer method and the other is shaker method. For the shaker method, the exciter must be fixed on the aircraft but it was very difficult because of the space limitation. Compared with shaker method, hammer method is much more flexible. In this paper, the hammer method was used to tap the upper and lower bolt locations on the mount which connects the engine. The actual hammer tap locations were shown in Fig. 10 and Fig. 11.

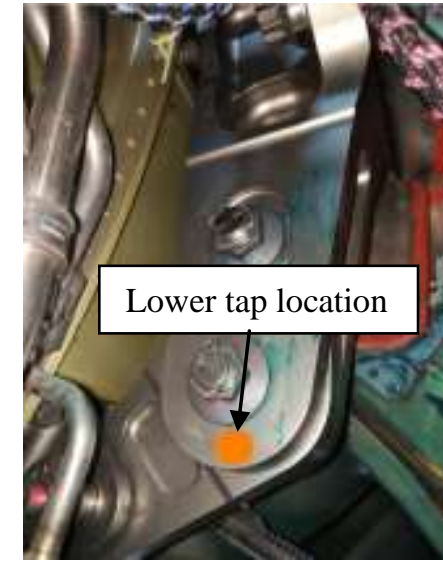

Fig. 10: Lower tap location on the mount

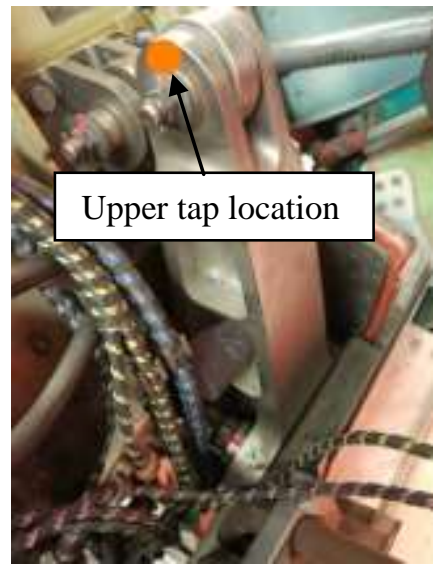

Fig. 11: Upper tap location on the mount

Two tri-axial accelerometers were installed on the pylon joint and pylon spar with fuselage as shown in Fig. 12 and Fig. 13. The two accelerometers have the same positions shown in Fig. 1.

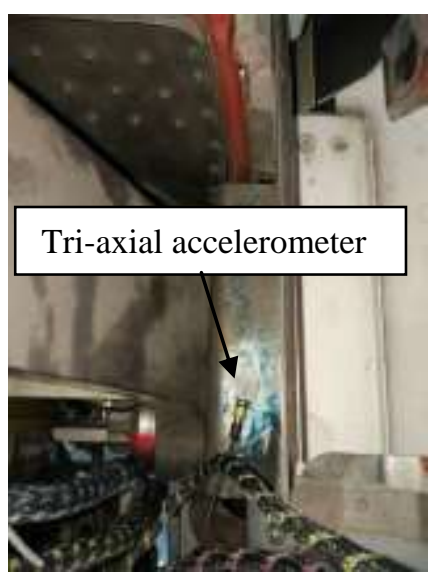

Fig. 12: Tri-axial accelerometer on the pylon joint

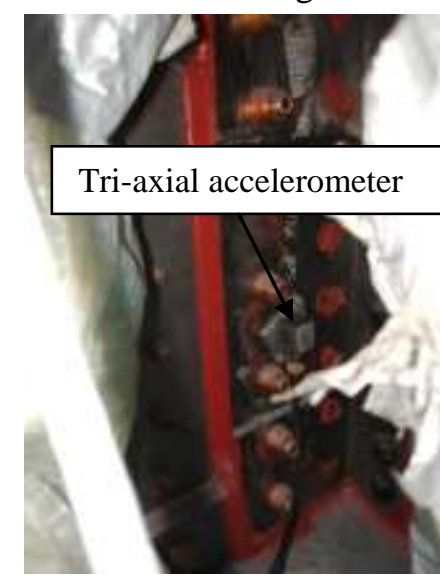

Fig. 13: Tri-axial accelerometer on pylon spar

\subsection{Experimental results}

Based on the tap test procedure, define the heading direction as $\mathrm{X}$ axis, lateral direction as $\mathrm{Y}$ axis and vertical direction as $\mathrm{Z}$ axis. Impact the lower location in $\mathrm{X}$ axis, repeat the measurement to obtain 5 averages FRFs between lower location $X$ direction and the response locations, then repeat the same measurement for the other two orthogonal axes to acquire FRFs between lower location $\mathrm{Y}$ and $\mathrm{Z}$ direction and the response locations. After the test on the lower location, repeat the same procedure for the upper location. The data analyzer can save all frequency-domain data automatically and compute the FRF for every direction after 5 averages impact. The FRF tap test results for the lower tap location and upper tap location with orthogonal directions of pylon joint acceleration response location and pylon spar acceleration response location are shown in Fig. 14 to Fig. 19 


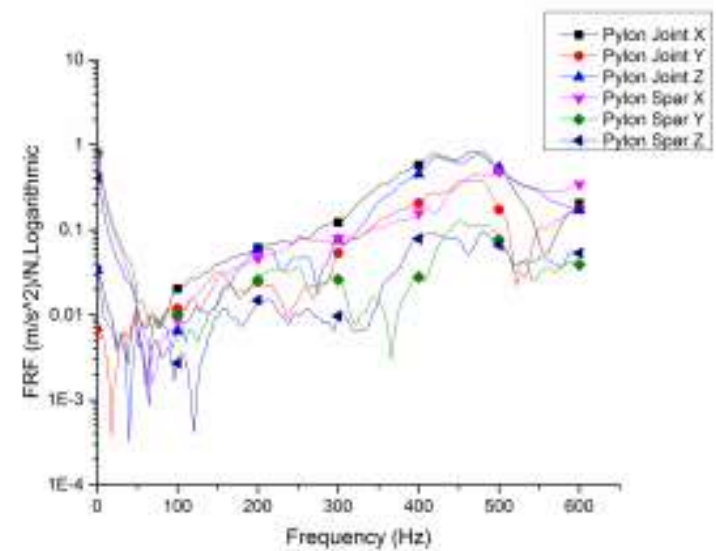

Fig. 14: FRF of lower tap location at $\mathrm{X}$ axis

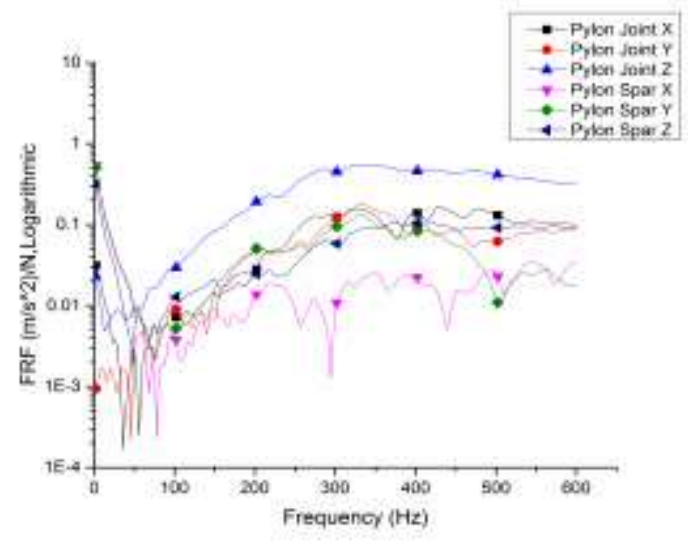

Fig. 16: FRF of lower tap location at $\mathrm{Z}$ axis

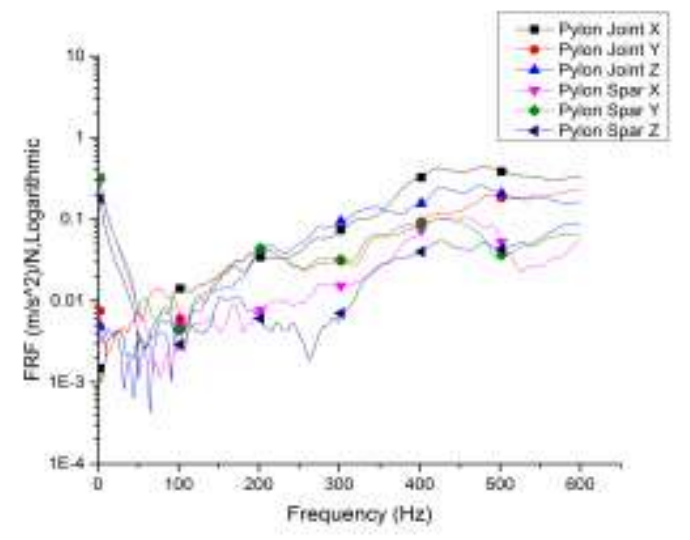

Fig. 18: FRF of lower tap location at $\mathrm{Y}$ axis

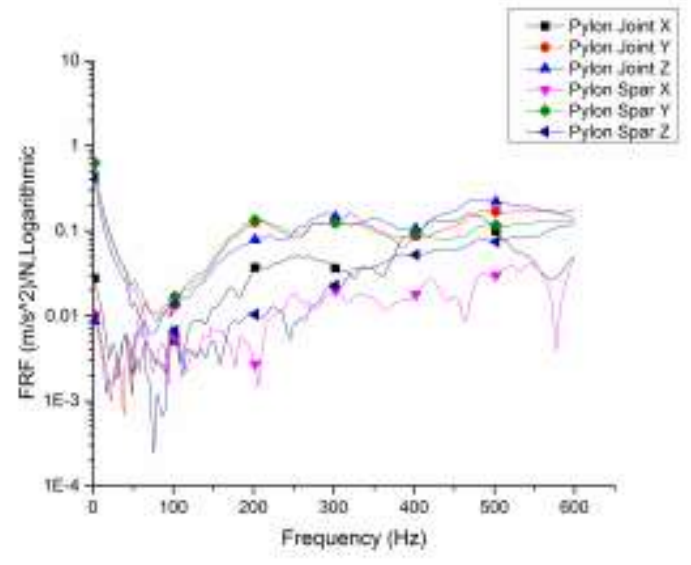

Fig. 15: FRF of lower tap location at $Y$ axis

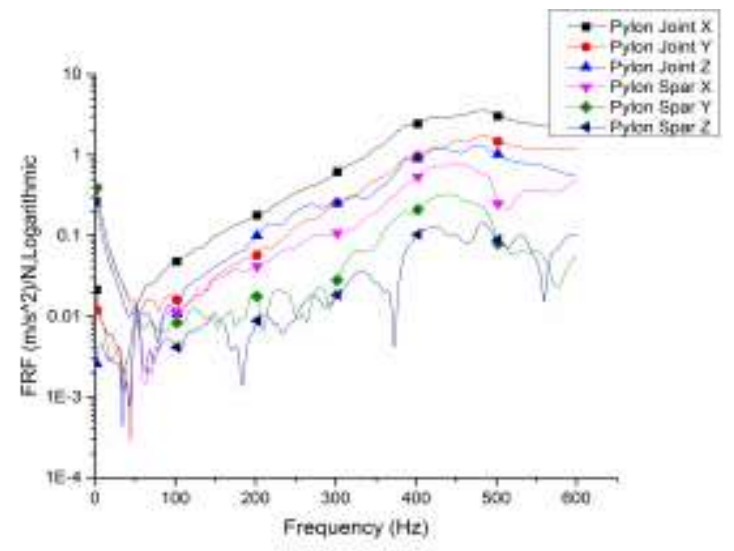

Fig. 17: FRF of upper tap location at $X$ axis

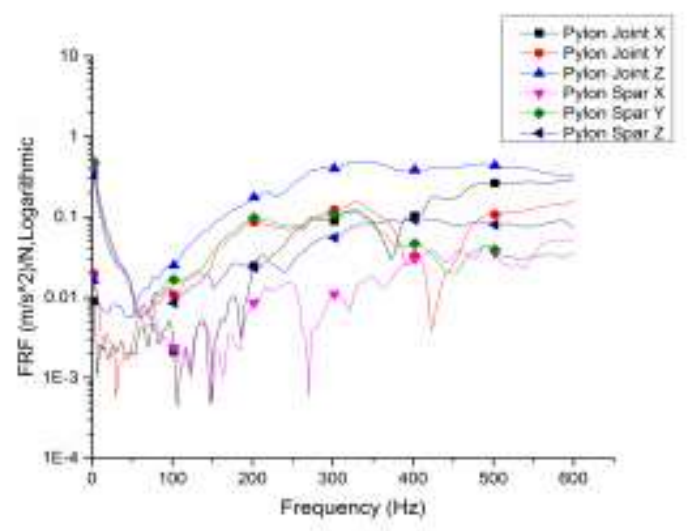

Fig. 19: FRF of upper tap location at $\mathrm{Z}$ axis

Compared with Fig. 4 to Fig. 9, it is clear that except the low frequency (below 50Hz), the prediction FRFs are in good agreement with the experimental results. Similar to the calculation results, there isn't any "peak area" for the fundamental frequency of N1 $88 \mathrm{~Hz}$ and N2 $270 \mathrm{~Hz}$ and for the upper tap location at X direction, the FRF value is actually very high from $400 \mathrm{~Hz}$ to $500 \mathrm{~Hz}$. For all experimental results, it was obvious that the values below $50 \mathrm{~Hz}$ were not accurate because of inherent flaws of hammer method, but it has no impact to the conclusions because the concerned frequencies were all more than $50 \mathrm{~Hz}$.

\section{Conclusions}

The most important result of this research is to validate the vibration transmission caused by engine low pressure rotor $(\mathrm{N} 1)$ and high pressure rotor $(\mathrm{N} 2)$ and make sure that the engine fundamental frequency vibration will not lead to obvious vibration and noise problem in the aircraft cabin. As a inherent characteristics of structure, structural FRFs were used to indicate this transfer behavior. 
In this work, the theory for the structural frequency response function were introduced and the numerical finite element model including the engine mount, pylon and pylon spar with fuselage was established. With the modal results of this finite element model and theory, the FRFS were calculated. To validate the calculated results, the hammer method was used to acquire the actual FRFs between the two excitation locations and the response locations of pylon joint and pylon spar with fuselage. Based on above research, the conclusions can be obtained as follows:

- For the commercial aircraft, it is an important work to evaluate the structural vibration transmission characteristics by structural FRFs. The concerned frequencies come from the vibration caused by engine operation in the cruise condition. These concerned frequencies can be calculated by the low pressure rotor $(\mathrm{N} 1)$ revolution speed and high pressure rotor $(\mathrm{N} 2)$ revolution speed.

- Based on this paper, the conclusion can be drawn that the calculated FRFs are in good agreement with the experimental results except the low frequency (below $50 \mathrm{~Hz}$ ).

- Numerical method can be used to predict the structural FRFs based on modal calculation results. But the structural damping parameters can't be defined precisely in the design phase so that it will lead to the calculation error to the test values.

- The method of hammer and shaker both can be used to acquire the structural FRFs. But in this paper, due to the space limitations, it was almost impossible to use the shaker method. For the hammer tap test, the results for the low frequency (less than $50 \mathrm{~Hz}$ ) were poor because of inherent flaws of hammer method.

- The numerical prediction of the structural FRFs was validated by experimental approach in this paper. Based on these frequency response function, it was confirmed that there was no resonant characteristics for the airframe structure itself at the concerned frequency of $86 \mathrm{~Hz}$ and $270 \mathrm{~Hz}$ and it may has no risk for the structure-borne noise caused by engine operation.

\section{Acknowledgements}

At the point of finishing this paper, I'd like to express my sincere thanks to my colleagues in Shanghai Aircraft Design and Research Institute. With the help of Luyao Ge belongs to the finite element team, the finite elements model was created quickly and submitted to the profession server for structure modes calculation. Secondly, I'd like to extend my thanks to Huayong Zhao and Feng Han, they were working with me about the hammer tap test on the aircraft so that I could get the experimental test results easily. Furthermore, the Flight Test Center belongs to COMAC also helped us to arrange the aircraft for test.

\section{References}

[1] Joseph P. Spagnol, Helen Wu, Chunhui Yang. Vibration Analysis of a Cracked Rotor with an Unbalance Influenced Breathing Mechanism. International Journal of Mechanical Engineering and Robotics Research. 2018,7(1):22-29.

[2] Chen Yi, He Erming, Hu xizhi, Han Feng. Exploring Wing-Mounted Engine Vibration Transmission for New Generation Airplanes with Turbofan Engines of High Bypass Ratio. Journal of Northwestern Polytechnical University. 2012, 30 (3): 384-388.

[3] Ewins D.J. Modal Testing: Theory and Practice. RSPLTD, 1986.

[4] Ward Heylen, Stefan Lammens, Paul Sas. Modal Analysis Theory and Testing. Katholieke Univ Leuven:Departement Werktuigkunde Press, 1998.

[5] Venkataramana Naik, G Prasanthi, D Sudhakara, and M Jayapal Reddy. Finite Element Vibration Analysis of PreStressed Functionally Graded Plates. International Journal of Mechanical Engineering and Robotics Research. 2012, 1(3):457-466.

[6] Fethi Kadığlu, Gülçin Tekin. Mixed Finite Element Formulation for the Free Vibration Analysis of Viscoelastic Plates with Uniformly Varying Cross-Section. International Journal of Mechanical Engineering and Robotics Research.2018, 7(3):235-239.

[7] Liu Dongming, Xiang Dang, Luo Qing, Zheng Jinxin. Applying Transfer Path Analysis to Automotive Interior Noise and Vibration Refinement and Development. Noise and Vibration Control. 2007, (4): 73-77. 